\title{
Repositorio Institucional
}

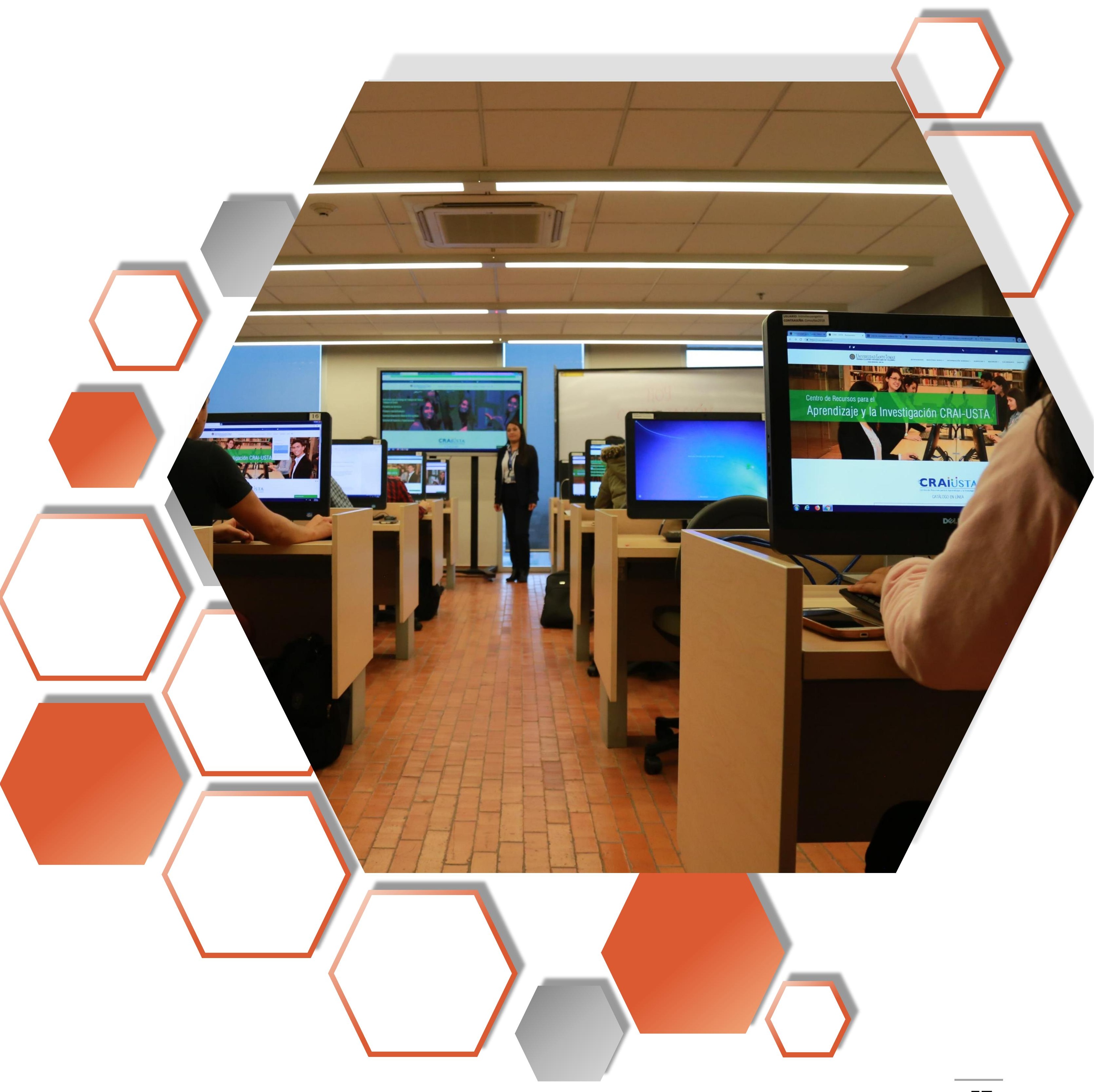




\section{Repositorio Institucional CRAI-USTA}

El Centro de Recursos para el Aprendizaje y la Investigación CRAI-USTA, inició con la implementación del Repositorio Institucional de la Universidad Santo Tomás en julio de 2015. El proceso fue retomado en el segundo semestre de 2016, donde se realiza un benchmarking con repositorios nacionales e internacionales y junto con el apoyo del proveedor, se aplica una serie de mejoras a nivel tecnológico, de socialización, articulación y visibilidad.

Con esta implementación se logró centralizar la producción institucional en una única plataforma de almacenamiento, estructurar las comunidades y colecciones en un entorno multicampus y mejorar la interfaz gráfica de manera que fuera funcional para mejorar la experiencia de los usuarios.

EI CRAI-USTA inicia con la tarea de contactar a cada uno de los departamentos y unidades de producción intelectual, con el fin de presentar el Repositorio Institucional, conocer sus necesidades, definir términos, acuerdos, requisitos y flujos de trabajo para ingreso de información y validaciones internas de cada unidad; posteriormente establecer los lineamientos del Repositorio Institucional, oficializarlos por medio de Acuerdo Rectoral y realizar la respectiva divulgación.

Para las tesis y trabajos de grado de los estudiantes de la Universidad Santo Tomás, se estableció un flujo de trabajo, a través de autoarchivo, que facilitó y agilizó el ingreso de información, dando la visibilidad de acceso libre a la producción académica de la Universidad.

En articulación con la Dirección de Investigación e Innovación Sede Principal, se generaron estrategias para que la información contenida en el Repositorio Institucional, obtuviera mayor visibilidad por medio de herramientas de métricas, enriquecimiento de metadatos, redes sociales y académicas, logrando posicionamiento frente a la comunidad de investigación nacional e internacional.

A continuación se presentan los resultados de este trabajo.

María del Pilar Florián Escobar ORCID 0000-0002-8977-7873

Diego Daniel Castillo Medellín ORCID 0000-0002-1159-900X 


\section{Ficha técnica}

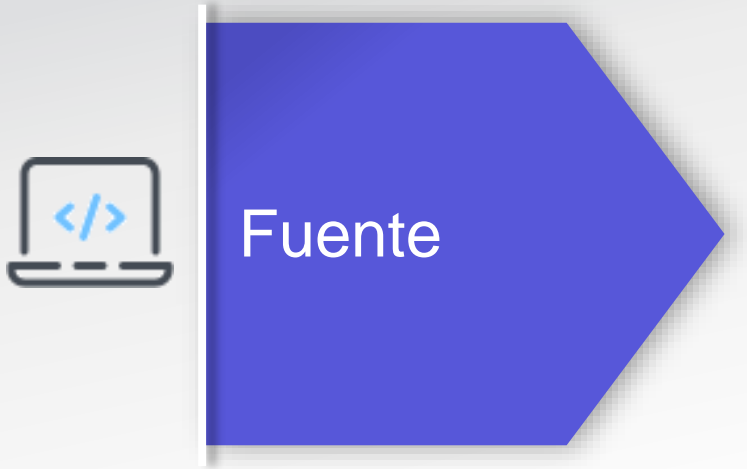

Google Data Studio

Expresión de búsqueda
Módulo incorporado en el Repositorio Institucional USTA DSpace 6.3

Publication repository.usta.edu.co
Harzing's Publish or Peris (POP), Microsoft Excel
(1)-0-0 Periodo de análisis

8 2015-2018

(i....) Más información

\section{DATOS USTA}

http://observatoriocienciometria.usta.edu.co/index.php/metricas -usta/autores-y-grupos-de-investigacion

\section{DATOS ABIERTOS:}

DOI: 10.5281/zenodo.3235623

Fecha de consulta: 24/01/2019 


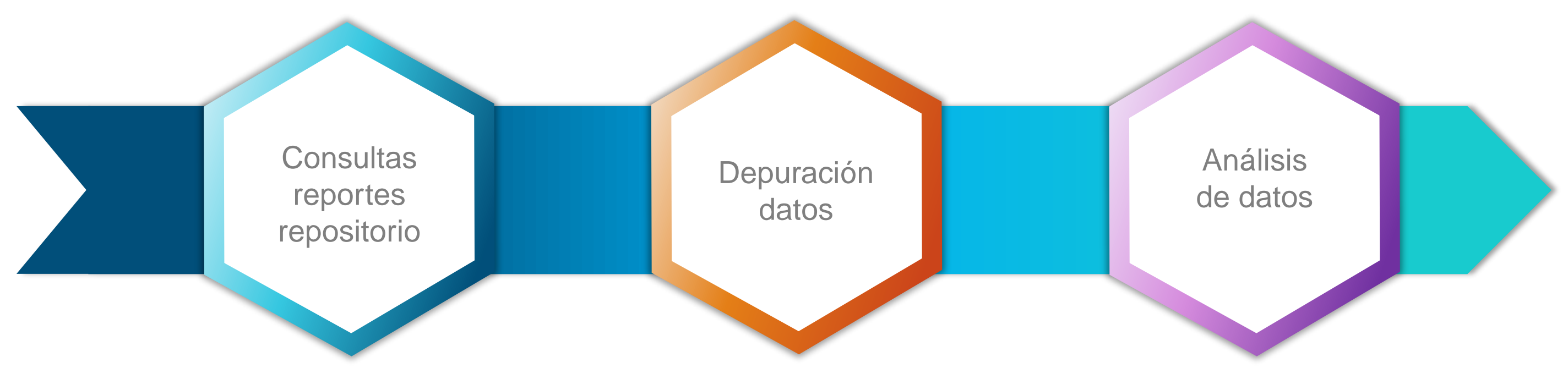

\section{Resultados}

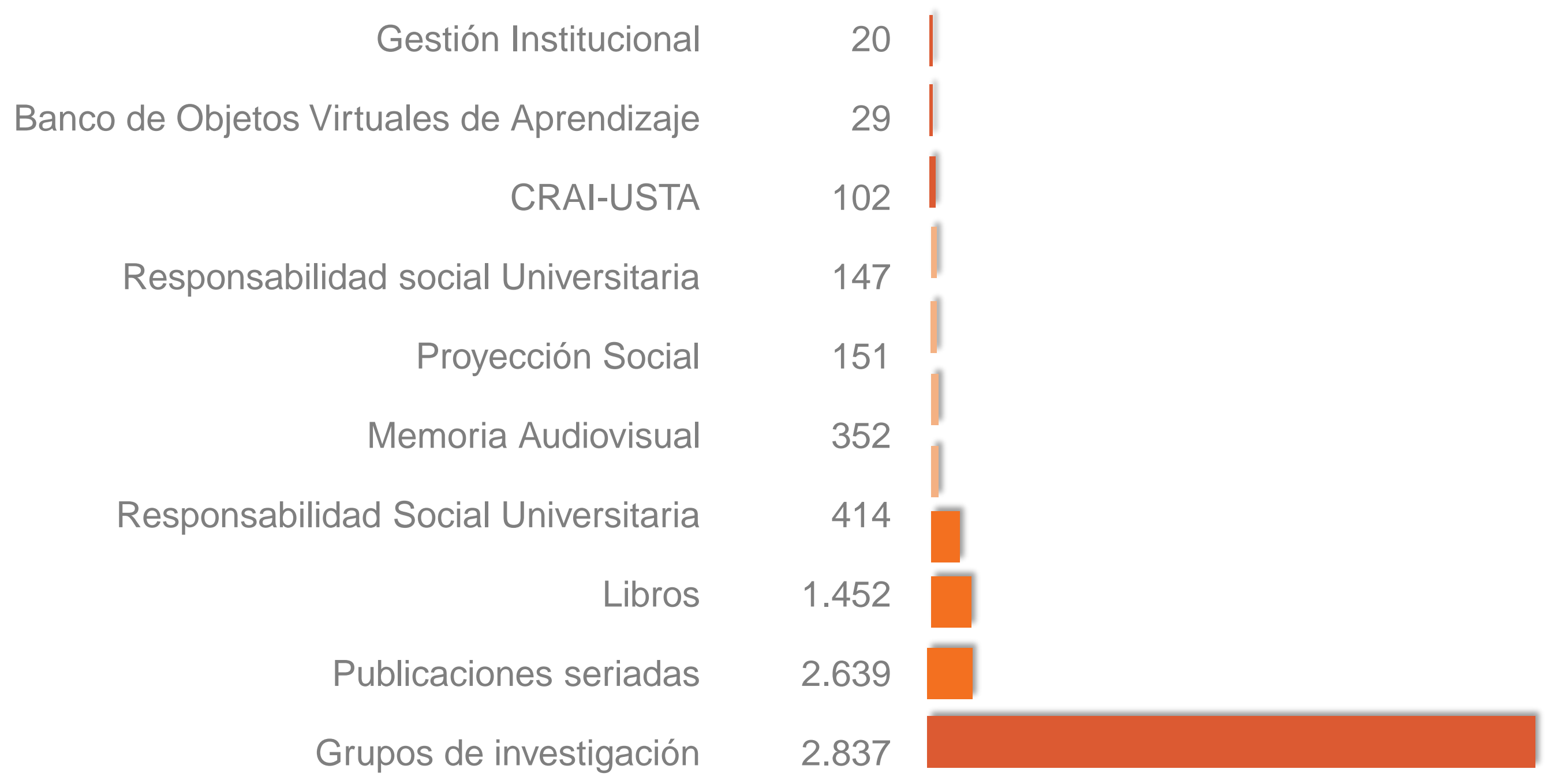

Tesis, trabajos de Grado y Disertaciones

41.420

Gráfica 32. Total de consultas por comunidad 


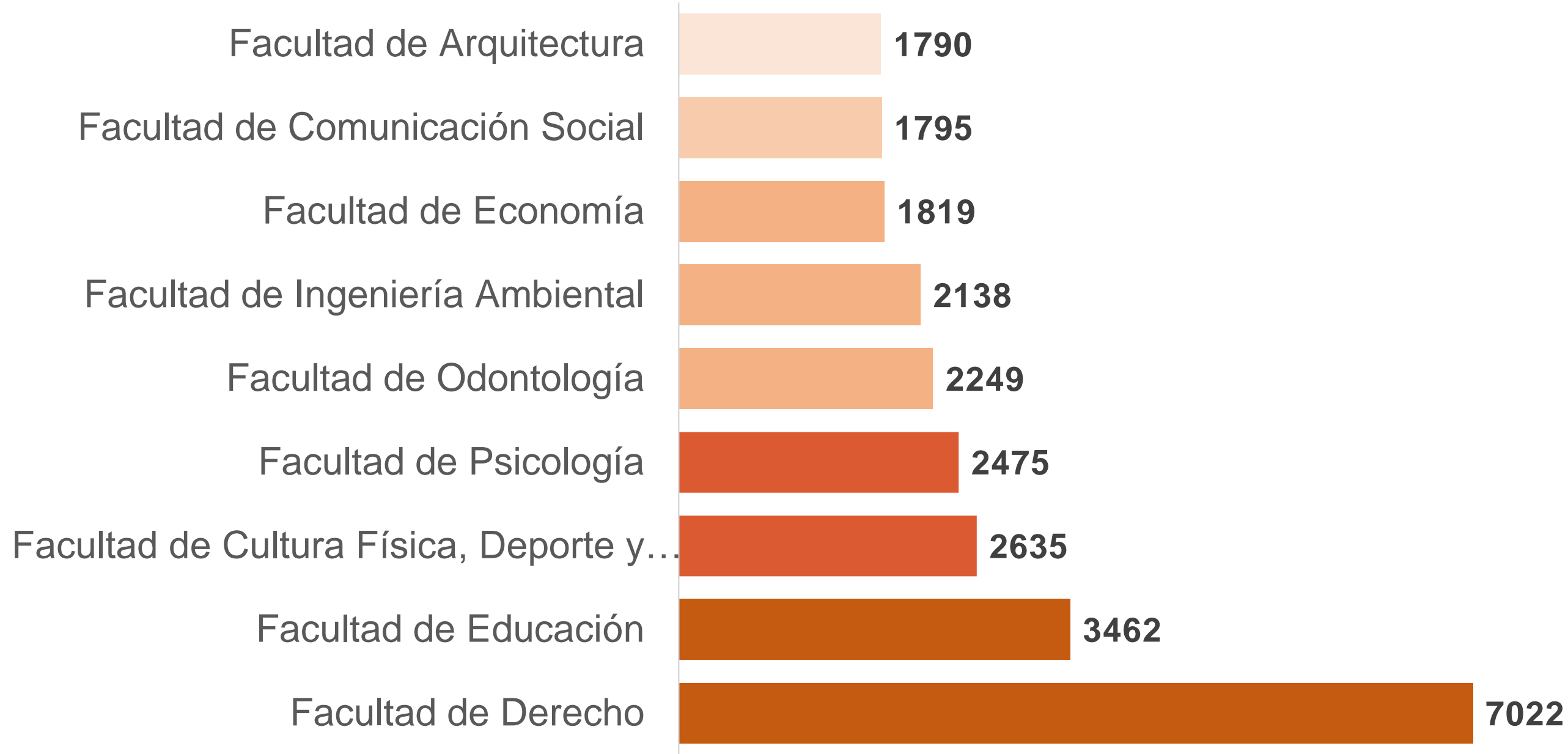

Gráfica 33. Total de consultas por subcomunidad

\subsection{4}
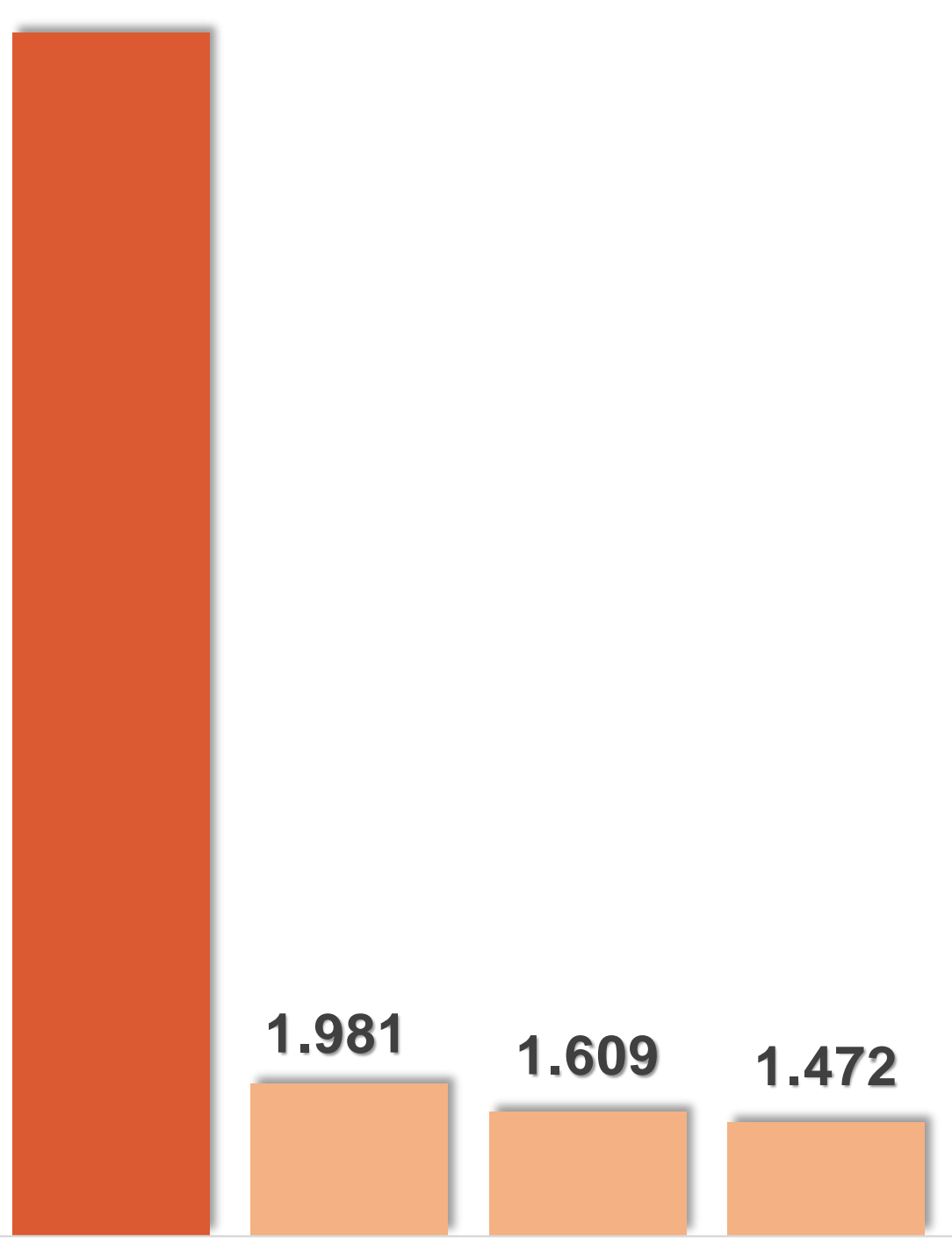

Bógota

Bucaramanga Villavicencio Floridablanca

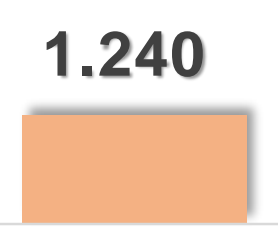

Medellín

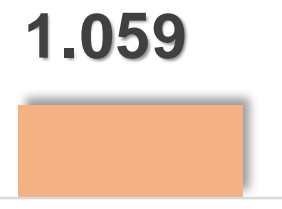

Cali

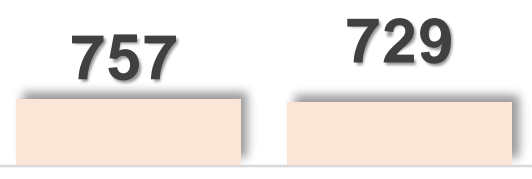

Barranquilla
592

Tunja
504

Cucuta

Gráfica 34. Ciudades con mayor consulta en el repositorio 


\section{Top 10 de los términos de búsqueda más}

usados

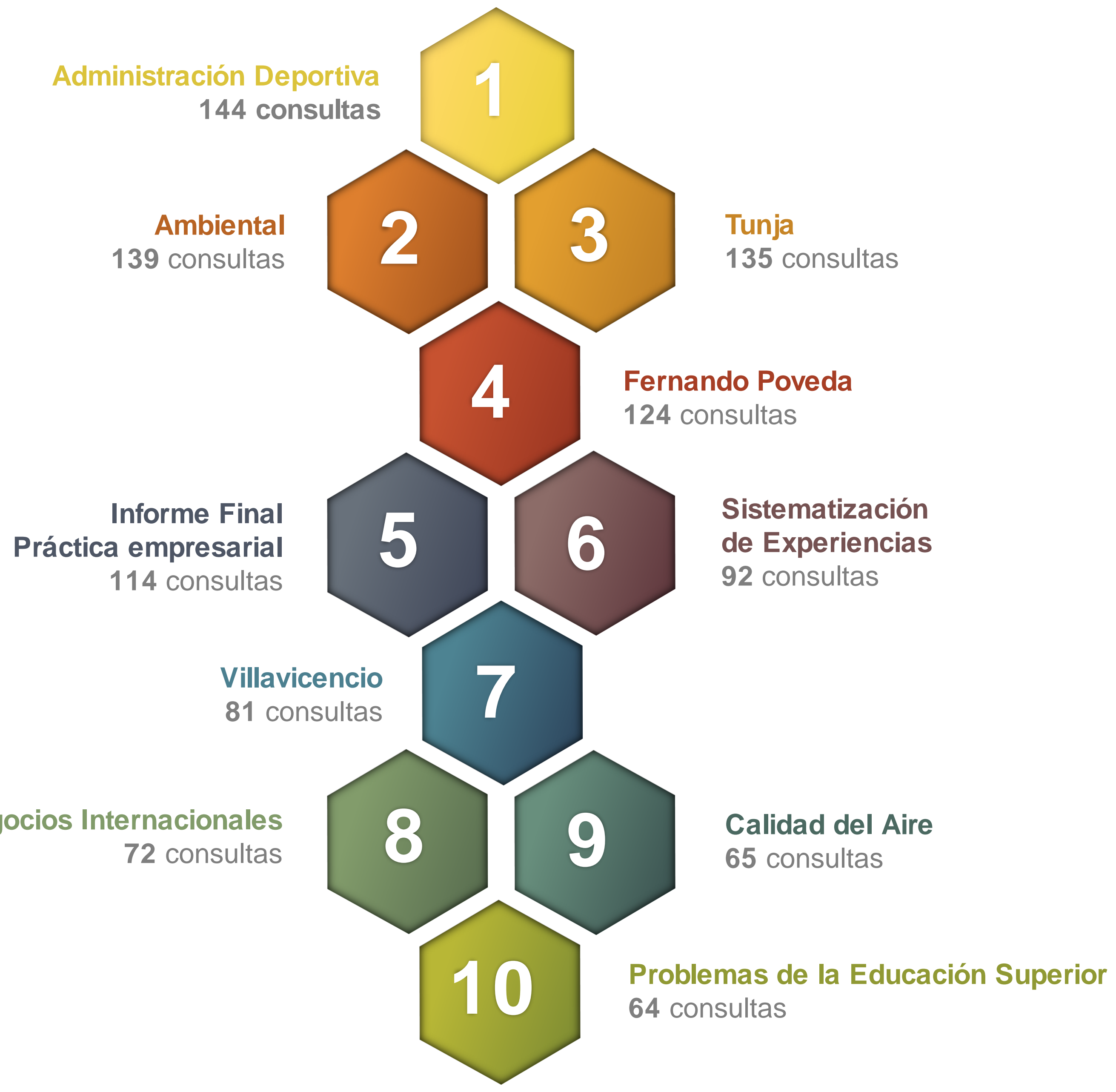




\section{Top 10 de los productos con más descargas}

1

DESCARGAS

187

2

DESCARGAS

3

DESCARGAS

141

146

Mejía Correa, Iván Fernando; Arciniegas Vega, Hernán Antonio

4

DESCARGAS

131

5

DESCARGAS

122

6

DESCARGAS

115

7

DESCARGAS

115

Gómez Serreto, José Guillermo

Diagnóstico del impacto del plástico - botellas sobre el medio ambiente: un estado del arte

Antropología filosófica - José ángel García cuadrado

\section{Jiménez Hernández, Juan de la Cruz}

Bullying en un contexto escolar masculino de la ciudad de Bogotá, Colombia

Corchuelo Rodriguez, Camilo Alejandro Modelos para la certificación de productos en la medición de Colciencias (Presentación)

Pérez Barrera, Ana María Feminicidio, la realidad en Colombia

Corchuelo Rodriguez, Camilo Alejandro Modelo Certificación - Consultorías e informes técnicos finales

Corchuelo Rodriguez, Camilo Alejandro Modelo Certificación - Participación ciudadana en proyectos de CTI

\section{Melo Bohórquez, Jazmín Johana}

La economía naranja de Bogotá: análisis de los aportes del sector de la publicidad

\section{DESCARGAS}

107

\section{9}

DESCARGAS

98

Lenis Barragán, María Paula; Torres Sanchez, Geisson Steeven

Documento de Sistematización de Experiencias: ¿Duros del diseño? Habilidades Socioemocionales en el Perfil del Diseñador

Corchuelo Rodriguez, Camilo Alejandro fomento a la CTI.docx 


\section{Bibliografía}

\section{$\mathbf{A}$}

Alperin, J. P., \& Rozemblum, C. (2017). La reinterpretación de visibilidad y calidad en las nuevas políticas de evaluación de revistas científicas. Revista Interamericana de Bibliotecología, 40(3), 231-241. Recuperado de https://doi.org/10.17533/udea.rib.v40n3a04

Arenas, J. L. de, \& Santillán-Rivero, E. G. (2002). Bibliometría ¿para qué? Biblioteca Universitaria, (1), 3-10.

\section{C}

Colciencias. (2017). Informe de la Convocatoria para Indexación de Revistas Científicas Colombianas Especializadas - Publindex. Recuperado de https://www.colciencias.gov.co/sites/default/files/upload/noticias/informe-publindex.pdf

Corchuelo-Rodríguez, C. A. (2013). Bibliometría: análisis del índice: los indicadores de autor y su aplicación en la comunidad científica colombiana (Trabajo de grado para optar por el título de Profesional en Sistemas de Información, Bibliotecología y Archivística, Universidad de la Salle). Recuperado de http://eprints.rclis.org/24678/

Corchuelo-Rodriguez, C. A. (2018). Factor de impacto. Recuperado de https://www.youtube.com/watch?v=QrfaTRyEAwo

\section{D}

Delgado López-Cózar, E., Orduña-Malea, E., Marcos Cartagena, D., Jiménez-Contreras, E., \& Ruiz-Pérez, R. (2012). JOURNAL SCHOLAR: una alternativa internacional, gratuita y de libre acceso para medir el impacto de las revistas de Arte, Humanidades y Ciencias Sociales.

Recuperado de http://digibug.ugr.es/handle/10481/20375 
Falagas, M. E., Pitsouni, E. I., Malietzis, G. A., \& Pappas, G. (2008). Comparison of PubMed, Scopus, Web of Science, and Google Scholar: Strengths and weaknesses. FASEB Journal, 22(2), 338-342. Recuperado de https://doi.org/10.1096/fj.07-9492LSF

\section{$\mathrm{H}$}

Hirsch, J. E. (2005). An index to quantify an individual's scientific research output. Proceedings of the National Academy of Sciences of the United States of America, 102(46), 16569-16572. Recuperado de https://doi.org/10.1073/pnas.0507655102

\section{L}

López, E. A., \& Arbeláez, E. J. V. (2016). Reapropiación del conocimiento y descolonización: el acceso abierto como proceso de acción política del sur. Revista colombiana de sociología, 39(2), 69-88.

\section{M}

Meho, L. I., \& Yang, K. (2007). Impact of data sources on citation counts and rankings of LIS faculty: Web of science versus scopus and google scholar. Journal of the American Society for Information Science and Technology, 58(13), 2105-2125. https://doi.org/10.1002/asi.20677 Pendón, F. C. (2015). Guías de la BUS: Índice h: en Google Scholar. Recuperado de https://guiasbus.us.es/indiceh/google scholar

\section{$S$}

Scimago. (2016). SJR - International Science Ranking. Recuperado de http://www.scimagojr.com/countryrank.php

\section{$\mathrm{T}$}

Túñez, M., \& Pablos, J. M.. (2013). El «índice h» en las estrategias de visibilidad, posicionamiento y medición de impacto de artículos y revistas de investigación. Recuperado de http://uvadoc.uva.es:80/handle/10324/2996 